\title{
TOGETHER ALONE: AN EXPLORATION OF THE VIRTUAL EVENT EXPERIENCE
}

\author{
OLIVIA WREFORD,* NIGEL L. WILLIAMS, $†$ AND NICOLE FERDINAND $\ddagger$ \\ *Business Growth \& Development Executive, Wasserman, London, UK \\ $\dagger$ Faculty of Business and Law, Portsmouth Business School, \\ University of Portsmouth, Portsmouth, UK \\ ‡Oxford Brookes Business School, The Oxford School of Hospitality Management, \\ Headington Campus, Oxford, UK
}

\begin{abstract}
After 50 years in development, virtual reality (VR) has now become commercially available to consumers. The events industry has started to adopt this transformational technology, by implementing it into live events or using it as an alternative method for providing event experiences. However, little research attempts to compare real to virtual event experiences to understand perceived user benefits and drawbacks. Using Uses and Gratifications (UG) Theory, this study aims to understand the possible user benefits provided from virtual event experiences. A process was designed that incorporated the viewing of a VR experience that was similar to an event previously attended by respondents. They were then interviewed and performed a product reaction card exercise to compare their experiences. Analysis of the data suggests that current 360 VR technology can be used to extend the experiencescape but not replace live events. Respondents indicate that VR provides emotional gratifications that may build positive associations with event organizations and brands. However, VR in its current form does not provide the social and sensory gratifications of live events. VR can therefore be used to deepen relationships with existing participants or encourage future participation at events.
\end{abstract}

\section{Key words: Virtual reality (VR); Virtual communitas; Tourism; Cocreation}

\section{Introduction}

The past decade has seen the reintroduction of virtual reality (VR) (Frew, 2016; Stein, 2016), frequently described as a method of cognitively transporting a user into a virtual environment
(VE) through interactions and immersive content (Marchiori, Niforatos, \& Preto, 2017). VR has a wide variety of applications in both the academic and commercial world, spanning from rehabilitation research (Howard, 2017) to fire evacuation design (Kinateder et al., 2014). The events 
industry has displayed a growing interest in VR, and organizations have started to incorporate this technology into planned events (Mintel, 2016a, 2016b, 2016c) such as the Consumer Electronics Show, Waze (http://app.sparksonline.com/companynews/sparks-waze-vr-highlighted-by-bizbash) product launch; the National Basketball Association (NBA) broadcast one game a week through VR (https://watch.nba.com/page/vr); and the Redbull Air Race (http://www.redbullairrace.com/en_ GB/simulator).

VR technology has progressed from the early Sensorama in 1962 through to the current headmounted displays (HMDs) (Stein, 2016), such as the Oculus Rift (https://www.oculus.com/) or HTC Vive (https://www.vive.com/uk/), headsets that block a user's peripheral vision to offer a wide field of view (FOV). HMDs are lightweight, comfortable and fully immersive (Pope, Dawes, Scheweiger, \& Sheikh, 2017), and can deliver two types of content, rendered (computer-generated imagery) and captured (filmed footage with a $360^{\circ} \mathrm{FOV}$ ), commonly known as three sixty-degree videos $\left(360^{\circ}\right)$ (Meinel, Heß, Findeisen, \& Hirtz, 2017).

HMDs have been used to display computergenerated imagery (CGI) as part of research into mental health issues (Dibbets \& Schulte-Ostermann, 2015), architectural planning (Maffei, Masullo, Pascale, Ruggiero, \& Romero, 2016), and physical rehabilitation (Carlozzi, Gade, Rizzo, \& Tulsky, 2013). HMDs have progressed further and can now deliver $360^{\circ}$ captured content (Barnes, 2017) from a real environment, which is deemed as more authentic in contrast to CGI VR (Pope et al., 2017). In the tourism domain, academics have used a Technology Acceptance Model perspective to determine the extent to which VR can influence visit intention to a destination (Huang, Backman, Backman, \& Moore, 2013) and a heritage site (Chung, Han, \& Joun, 2015). More recently, perceptions of presence and its impact on the virtual experience have been explored in tourism (Tussyadiah, Wang, Jung, \& tom Dieck, 2018) and events (tom Dieck, Jung, \& Rauschnabel, 2018). Getz (2016) asked, "Will virtual reality replace live event experiences?” (p. 172), which implies that the users could obtain the same gratifications from virtual and live experiences. This study proposes to investigate this issue by identifying user gratifications obtained from a $360^{\circ}$ VR event experience.

\section{Literature Review}

The event experience has been defined as an attendee's interactions with purposefully planned activities (Chen, Singh, Ozturk, \& Makki, 2014). It has been conceptualized as transformative as these experiences takes participants out of everyday routines (Pine \& Gilmore, 1998) using spatial and temporal arrangement of event elements (Richards, Marques, \& Mein, 2014). Others have proposed that social interaction defines the event experience (Arnould \& Price, 1993) as the coming together of attendees or the "communitas" of an event (Morgan, 2008). These interactions can result in unplanned (Lee, Brown, King, \& Shipway, 2016.), beneficial emergent (sensory or behavioral), or sentiment outcomes for attendees (Geus, Richards, \& Toepoel, 2016). In the former domain research has identified the sensory and physical aspects of the event experience (Getz, 2008, 2012). In the latter domain, research has identified affective (Chen et al., 2014) and cognitive (Ayob, Wahid, \& Omar, 2013) outcomes. While event attendance can stimulate the development of social identities (Schmitt, 2003), the presence of others is not only positive. Social interactions at events can also stimulate negative reaction and the company of others can contribute towards feelings of insecurity and fear (Nordvall, Pettersson, Svensson, \& Brown, 2014).

Traditionally, event activities are staged in a physical environment that provides the framework or eventscape in which activities and interactions occur (Ferdinand \& Williams, 2018). However, recent developments in technology present a simulated alternative. VR, as described by Bates-Brkljac (2012), is the process of replacing "real images, sound, and tactility with computer-generated illusions” (p. 52) to create a virtual environment (VE) for users to navigate in and interact with. Research suggests that, like events, VR can also stimulate physical and emotional responses (Meinel et al., 2017). Recent developments in technology now allow users to experience real-life videos through $360^{\circ}$ filming and live streaming; $360^{\circ}$ is the use of special camera systems that capture a real-world scene in all directions and can be delivered through multiple VR devices (Meinel et al., 2017). Event locations could be replicated through $360^{\circ} \mathrm{VR}$, and 
the event experience could be simulated (Marchiori et al., 2017). VR has been indicated as a useful tool for marketing tourism experiences as they are difficult for customers to evaluate without interaction (Wan, Tsaur, Chiu \& Chiou, 2007).VR may perform a similar role for events that have been captured in a $360^{\circ}$ format, from sport events (https:// www.youtube.com/watch?v=BPPhuPgIp6g), to corporate functions (https://www.youtube.com/ watch?v=1ji220_ULAU\&t), to festivals (https:// www.youtube.com/watch?v=j81DDY4nvos).

\section{VR Versus Live Events Tensions}

The recent reduction in the cost of VR headsets have enabled access to a larger group of interested users seeking to view events in this format (Slater \& Sanchez-Vives, 2016). Finally, VR content can now be distributed on social media platforms such as YouTube and Facebook, increasing availability of content (Nasrabadi, Mahzari, Beshay, \& Prakash, 2017). Related work has suggested that while the mediated live events experience can theoretically provide sensation and social benefits (Mueser \& Vlachos, 2018), these benefits have not been empirically verified in VR content.

\section{Uses and Gratifications of VR Events Content}

VR research in related areas has been criticized for being atheoretical (Yung \& Khoo-Lattimore, 2017). When theory has been used, it has adopted frameworks such as the previously mentioned Technology Acceptance Model and the Theory of Planned Behavior (Han, Yoon, \& Woods, 2016) to examine motivations to use VR technology. By capturing a live experience, VR may be a type of recorded media. Uses and Gratification (U\&G) theory developed from observations of user interactions with mass media and seeks to understand the benefits or gratifications derived from these interactions (Lichtenstein \& Rosenfeld, 1983). Media content can provide cognitive gratifications by enabling a user to increase their understanding of a given issue. Emotional gratifications, including escape from daily routines, can also be obtained from interacting with media content. Beyond individual uses (Swanson, 1992), media can meet relational needs such as increasing a users' status with a given group (Karapanos, Teixeira, \& Gouveia, 2016).

The U\&G approach has been criticized as it does not consider institutional logics such as external, macrolevel political, social, or cultural factors that may influence perceptions of particular media sources and, hence, the benefit derived from them (Papacharissi, 2009). While digital technology platforms such as social media can extend the gratifications provided by mass media to include pastime, affection, fashion, sharing (Quan-Haase \& Young, 2010), virtual community, and mobility (Sundar \& Limperos, 2013), the costs of these benefits are not fully understood. However, the U\&G focus on individual users' interactions enables examination of benefits derived from both medium and format characteristics of technologies such as VR (Haridakis, 2013).

VR provides additional possibilities for gratifications based on navigability or the ability to control the perspective from which content is experienced (Sundar, 2008). This enables VR to provide physical, emotional, and cognitive gratifications (Murray, Neumann, Moffit, \& Thomas, 2016) along with immersion (being there) and presence (Sundar \& Limperos, 2013). In VR research, emotional gratification is a common subject area and has been investigated across a variety of industries (Dibbets \& Schulte-Ostermann, 2015; Kwon, Powell, \& Chalmers, 2013; Serrano, Botella, Baños, \& Alcañiz, 2013). Using VR, emotions such as joy, sadness, boredom, anger, and anxiety have been successfully elicited (Falconer et al., 2014).

Immersion is described as the feeling of being inside a VE, enabling the user to escape from the real world in a manner that differs from viewing content on other media (Rebelo, Noriega, Duarte, \& Soares, 2012). HMDs with headphones provide the highest level of immersion in commercially available systems (Pope et al., 2017). A related aspect is presence, where a user is consciously aware of being in a VE (Gutierrez, Vexo, \& Thalmann, 2008) and can navigate within the experience. While presence can be influenced by the technological aspects, the nature of VR content will also influence a user's sense of presence (Peperkorn, Diemer, \& Mühlberger, 2015). Unlike other media, VR can provide physical gratifications and has been used as a therapy tool that 
stimulates muscle development (Kilteni, GrauSánchez, Veciana De Las Heras, Rodríguez-Fornells, \& Slater, 2016). These gratifications are summarized in Figure 1.

Recent literature has used VR to compare a user's behavior, interactions, and perception of a real world and a corresponding CGI virtual world (Maffei et al., 2016). To date, the types of gratifications provided by VR event and festival content is not yet known. This research seeks to identify the gratifications obtained by viewers of VR content by comparing a user's perceived experience of a realworld event to a $360^{\circ} \mathrm{VR}$ simulation of an event type that they had previously attended.

\section{Research Method}

A flexible qualitative approach was used to collect data that incorporated a viewing of VR content that was similar to an event that the respondent attended in the past with interviews and a product reaction card test. The method was influenced by the Karapanos et al.'s (2016) approach, which examined users' memorable experiences on social media. This approach encourages participants to focus on a specific experience that attempts to minimize recall bias by enabling users to compare perceptions of the past and present activity (Sheldon, Elliot, Kim, \& Kasser, 2001). Respondents were recruited using chain referral sampling to identify respondents with specific characteristics from a subset of the general population. Individuals were recruited from London

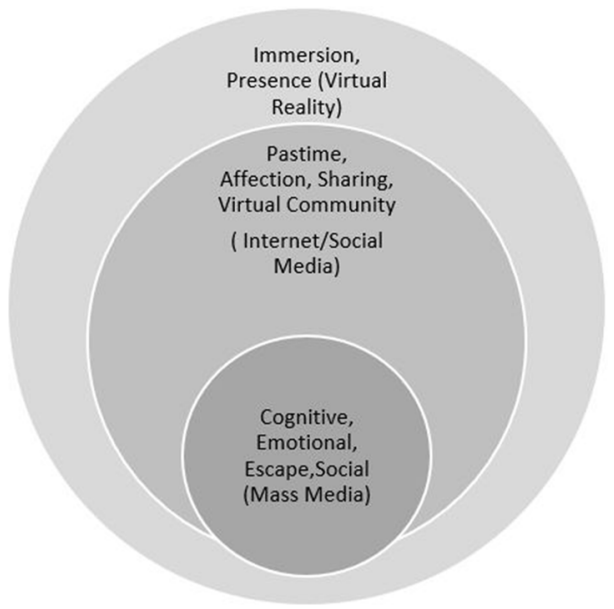

Figure 1. User gratifications from media types. event organizations that met one of the specific criteria (Palinkas et al., 2015): 1) I have been to a live NFL game; 2) I have been to a live semiprofessional or professional basketball game; 3) I have been to a concert and like the band Kasabian.

Ten interviews were conducted over the course of 2days (February 15-16, 2017), with the protocol described in Table 1. Each semistructured interview had a duration of 45-60 min to explore perceptions of VR. These interviews incorporated a product reaction card (PRC) activity, an approach designed to facilitate the immediate comparison of products (Benedek \& Miner, 2002). This approach was used as an activity to compare the VR event experience to the recall of an event experience, to gain insights into the intangible aspects of the user's virtual and real event experience. The Benedek and Miner (2002) tool kit contained original set of 118 words, which was reduced to a set of 55 to focus on words had relevance to either the real and/ or virtual event experience. This methodology was previously to compare the user experience of a real building and a corresponding virtual model (Kuliga, Thrash, Dalton, \& Hölscher, 2015). After being exposed to the VR event experience, participants were asked to choose five words from, but were not limited to, the modified list. Each word was then discussed, allowing for further understanding of the rationale behind each choice. This activity was repeated when the topic of conversation moved onto the recall of an event experience. The words were then formatted into a diagram depicting all words that were chosen to describe both event experiences.

In the findings below, the participants have been referred to by the following codes: P1 (Office Manager), P2 (Account Manager), P3 (Operations Director), P4 (Senior Designer), P5 (Head of Talent), P6 (Digital Director), P7 (Jr Project Director), P8 (Jr Account Director), P9 (Account Manager A), P10 (Account Manager B). With permission from the participants, the interviews were audio recorded and transcribed (Richards, Marques, \& Mein, 2015) (see Fig. 2).

\section{Data Analysis}

First, the terms selected from the PRC activity such as "appealing," stimulating," and "unique" were summarized using a diagram (Fig. 3). Next, 
Table 1

Research Protocol

\begin{tabular}{|c|c|}
\hline Minutes & Details \\
\hline $0-5$ & $\begin{array}{l}\text { Short introduction, including details of whole research project } \\
\text { Participant signs consent form confirming ability to participate in VR experience }\end{array}$ \\
\hline 5-15 & $\begin{array}{l}\text { Participant is exposed to a short } 360^{\circ} \text { VR event experience on HTC Vive } \\
\text { - Michigan Gameday in VR (https://www.youtube.com/watch?v=B30AmPhRXxo\&t) } \\
\text { - Basketball experience (https://www.youtube.com/watch?v=wq6a7ncMugQd } \\
\text { - Shrillex Live at Electric Daisy Carnival Brazil https://www.youtube.com/watch?v=3uhmA5dCk40 } \\
\text { Participant is led into interview room }\end{array}$ \\
\hline $15-25$ & $\begin{array}{l}\text { Opening questions } \\
\text { Introduce the product reaction card activity for the VR event experience } \\
\text { Product reaction card activity is completed } \\
\text { Participants describes why they chose each word }\end{array}$ \\
\hline 25-35 & $\begin{array}{l}\text { Additional } 5 \text { questions were asked in relation to the VR event experience } \\
\text { Closing conversation about VR }\end{array}$ \\
\hline $35-45$ & $\begin{array}{l}\text { Introduce the Product Reaction Card activity for the recall of an event experience } \\
\text { Product Reaction Card activity is completed } \\
\text { Participants describes why they chose each word }\end{array}$ \\
\hline $45-55$ & $\begin{array}{l}\text { Additional } 5 \text { questions were asked in relation to the recall of an event experience } \\
\text { Closing conversation about the event experience }\end{array}$ \\
\hline $55-60$ & Final comments and clean up questions \\
\hline
\end{tabular}

Note. HTC Vive headset specifications: Model 0PJT100, including SteamVR Tracking 1.0 technology. Computer components to power the HTC Vive: (1) Operating system: Windows 10 Home 64-Bit; (2) CPU: Intel Core i7-6950X; (3) Graphics card: EVGA GeForce GTX 970 x2 (SLI option).

the text of the interviews was transcribed and coded (Braun \& Clarke, 2013). Using an iterative approach, an open coding approach was first applied to the text of transcriptions of user's perceptions of VR (Silver \& Lewins, 2014).
The codes were then examined in an iterative manner and clustered into related groups. These code groups were then classified by comparison with the literature on user gratifications into themes (Table 2). These themes were classified into

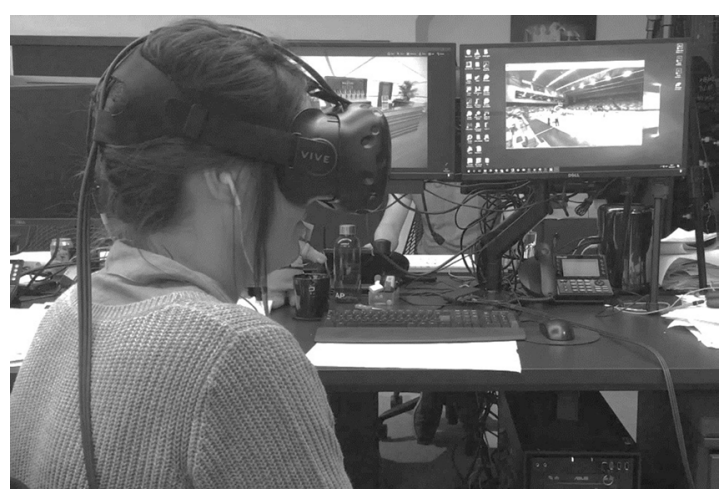

Figure 2. Participant during the VR experience (personal collection). 


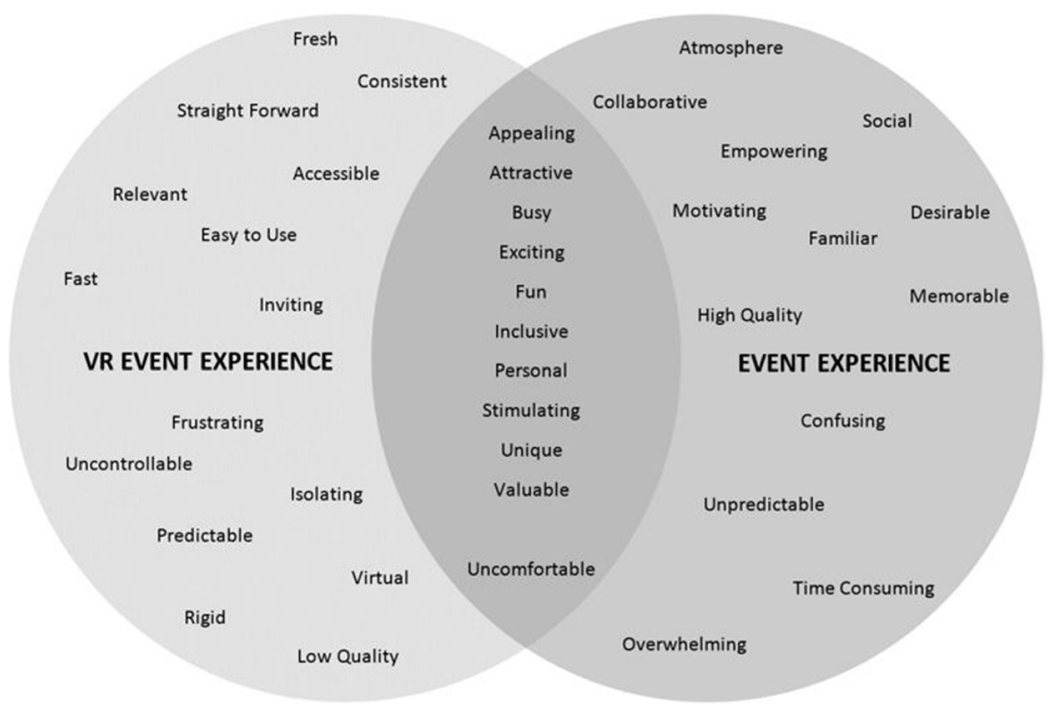

Figure 3. Product reaction card.

gratifications provided by the VR experience and gratifications desired by customers that were not provided by the VR experience.

\section{Theme Elaboration}

\section{Emotional Memory Gratification}

Liu, Sparks, and Coghlan (2016) acknowledged a variety of positive and negative emotions that could be experienced at events. From the product reaction task, VR event experience was seen to generate feelings of excitement (appealing, exciting, fun) in a similar manner to a live event (Howard, 2017):
Having past experiences is beneficial because you can draw upon those real life experiences, but you also compare it. (P10)

VR has been previously found to evoke past memories (Freeman et al., 2014) and this research extends this finding to indicate that VR can encourage users to recall past event experiences.

\section{Autonomous Immersion}

Immersion and presence are heavily interconnected (Peperkorn et al., 2015) and informants stated that the VR event experience drew the user's attention. Further, users were able to autonomously access the experience without significant

Table 2

Virtual Reality (VR) Event Experience Gratifications

\begin{tabular}{|c|c|}
\hline Gratification & Indicative Product Reaction Card Terms \\
\hline \multicolumn{2}{|l|}{ Gratifications provided } \\
\hline Emotional memory stimulation: Sensations evoked by VR & Stimulating, exciting, fun, busy \\
\hline $\begin{array}{l}\text { Autonomous immersion: Ability to access and } \\
\text { control immersive experience }\end{array}$ & $\begin{array}{l}\text { Accessible, attractive, personal, easy to } \\
\text { use, relevant, valuable }\end{array}$ \\
\hline Newness: New experience for the first time & Fresh, unique, appealing \\
\hline \multicolumn{2}{|l|}{ Desired gratifications } \\
\hline $\begin{array}{l}\text { Virtual communitas: Social feeling invoked by } \\
\text { virtual experience }\end{array}$ & Inclusive, inviting \\
\hline Sensory realism: Multisensory experience of events & Low quality, isolating, uncomfortable \\
\hline Perspective realism: Realistic experience perspective & Rigid, predictable, virtual, frustration \\
\hline
\end{tabular}


intervention from staff. The PRC task identified words such as accessible, attractive, personal, easy to use, relevant, valuable. Users suggested that the $360^{\circ}$ footage supported the immersion process:

The real footage is more stimulating then computer generated footage, because it is actually like you are there so it makes it more realistic. (P1)

All informants acknowledged that they were engrossed in the VR experience, which confirms Barnes' (2017) description of $360^{\circ}$ as absorptive:

I think it feels quite natural that when they score everyone cheers. But then the other pieces of entertainment, like kiss cam, you are obviously queued to react in a certain way. (P8)

The VR content was perceived as stimulating and exciting; the $360^{\circ}$ was described as representative of real life:

It was wonderful, and also the music and the content (in the VR), was very much what I saw when I went to the Isle of Wight Festival. (P5)

The themes below are desired gratifications identified from the analysis that were not provided by the VR experience.

\section{Virtual Communitas}

At live events, interviewees felt connected to the people around them, since they were sharing and enjoying the same experience with like-minded people (Getz \& Robinson, 2014):

You feel a bit like there is a sense of tribe behavior. ... You have your side, you have your team, so you felt that sense of community, and that inclusive nature. (P8)

This tribal behavior is a key indicator of communitas (Malgorzata, 2014) and crowds can create positive atmospheres at music festivals. Informants felt that being in a crowd offered them opportunities for socialization and enjoyment; however, crowds were also described as unpredictable. Informants did not feel any such connection to the temporary community in the VR:

$50 \%$ of attending events is attending them with the people you share them with. When you are in a VR experience ... you don't have anyone to share it with. I think it's really hard to feel you are connected to anyone else. (P2)

This suggests that despite the immersion, VR did not simulate a sense of belonging or communitas.

\section{Physical}

During the VR viewing, discomfort or nausea was experienced as a result of the user's inability to control the pace or focus of the experience (Stein, 2016), which is a common and unfavorable impact of VR (Treleaven et al., 2015).

I did feel slightly nauseous to begin with, but I think it's when the camera moves off the coach and then pans around to go into the stadium. (P3)

Interestingly, the word "uncomfortable" was selected for both live events and VR. For events, a respondent indicated:

The crowd got busy and busy as we went in, but the music wasn't uncomfortable, it was more the setting, there are mosh pits that go on, so we soon came out.

\section{For VR:}

Uncomfortable, because it made me feel car sick. Maybe I feel travel sickness quite easily any way, but I think because I spent so much time spinning around because I wanted to look at everything, that was the only thing that was uncomfortable about it though for me. The headset isn't uncomfortable.

In the first case, discomfort arose from the potential for collisions with other attendees. In the second case, physical discomfort was induced from the user's eagerness to explore a VR experience. This finding indicates that physical comfort is sought not just in the equipment but in the delivery of the experience.

\section{Sensory Realism}

Informants found that the VR audio was described as inconsistent to real life sound. This could be a limitation of the recording equipment, the audio type, the headphones, or a combination of these (Lelyveld, 2015): 
I think it lacks that bass and reverberations that you associate with sound. Although the sounds sound the same, they didn't feel the same. Because you can hear people stomping, but you would also feel them stomping. That's the only difference. They were realistic sounds, but if I was in the room I would be experiencing them differently. (P8)

Martins, Gonçalves, Branco, Barbosa, and Melo (2017) recognized that audio is as important as video; therefore, this could be a deficiency in the VR event experience. Overall, the VR event experience could be acknowledged as stimulating for the eyes and ears, but in did not match the multisensory event experience.

\section{Perspective Realism}

In addition to sensations, the perspectives presented were not viewed as realistic. Some informants felt that the perspectives were unnatural for a normal consumer, as the content was filmed in places that an attendee would not access:

Where you are in the tech position ... you're not in the busiest area. It's not where you would choose to be naturally at a concert. . . . It cuts the realism a little bit, because if you have never worked in that space then you would never end up in that position. (P10)

A main goal of VR as the creation of believable environments (Disztinger, Schlögl, \& Groth, 2017); therefore, a lack of realism could be a flaw in the VR event experience. It is interesting that an attempt to provide a superior experience (perspectives not available to the average attendee) reduced the perceived realism and hence presence and immersion of the experience. Interestingly, participants acknowledged health and safety, and risk as significant components of the event design (Bladen, Kennell, Abson, \& Wilde, 2012) and viewed restrictions in real world events positively:

The general area had free roaming which gave people more experiences and more freedom to buy and enjoy ... restrictions were only there for people’s safety. (P5)

The PRC task identified words such as "predictable" and "rigid," suggesting that viewers felt that they were not part of the process. A passive experience gives users little control as they are taken on a storytelling journey (Lelyveld, 2015):

But I did really feel restricted because you are passively watching. (P4)

While VR was able to deliver exclusive content that would not be accessible to event attendees, some informants became frustrated with the predetermined views. They suggested that VR experience did not permit the same extent of freedom experienced at the real event:

Not being able to change the things that you're looking at can be quite frustrating. I wouldn't say it lessened my experience because it was so short, but if I was watching a whole game for example and it was stuck on that one bird's-eye view I think I might get frustrated. (P2)

Other informants indicated that the absence of a narrative made it hard for them to connect to the people in the content:

It's always really nice to have a narrative. As a snapshot, it works really well, but if you wanted to have any deeper attachment . . . a storyline it would have been better. (P3)

\section{Discussion}

While technology has been recognized as a threat to the events industry (Getz, 2016), delivering event experience through VR has certain limitations, which currently may influence the virtual event experience. This could be a consequence of the present technological boundaries of VR models (Rebelo et al., 2012), as well as a deficiency of research on the consumer experience of VR head-mounted displays (Gugenheimer, Wolf, Hass, Krebs, \& Rukzio, 2016). Subsequently, $360^{\circ}$ VR event experiences are mainly described to have an absence of sense stimulation and communitas, which are vital elements to an event experience (Berridge, 2015).

UG theory was a useful approach to understanding user perceptions of the virtual event experience. UG has been used to examine how users obtain emotional gratifications from media along with escape from real life. This research extends the UG body of knowledge to new gratifications obtained by users from an event VR experience. Current UG research 
does not consider the social gratification of communitas. Users in the study found it difficult to build attachments in VR as social interactions go beyond sight and hearing (Lee et al., 2016). This social sensation had both a physical and mental component. Informants mentioned that they could feel physical movements at real events as well as sensing the social presence at live events, something that the VR experience did not provide. The VR event experience could be perceived as an individual immersive content-led experience, which required passive involvement and had an absence of rich sensory stimulation, which is perceived as more realistic (Martins et al., 2017).

Previous research on VR identified that a narrative could contribute towards the authentic and immersive nature of a VR experience (Aylett \& Louchart, 2003). However, the guided or directed approach of content delivery used by designers of VR experiences was not viewed positively by users. Compared to a live event, there were no moments of discovery or serendipity that may enhance the event experience. In addition, while existing research identifies the impact of technology on the quality of the VR experience, research has not examined the need for perspective realism. VR has also been positioned as a means to provide access to perspectives that a typical attendee could not obtain. However, that potential advantage was not viewed positively by respondents and, paradoxically, reduced the perceived realism of the experience. For events, constraints such as security and viewing angles contribute to the realism of the experience. VR that dispenses with these constraints may be viewed as unnatural by observers.

However, VR can be used to extend the eventscape. Unlike livestreaming, which is simultaneous, VR offers opportunities for reflection on past events that may not be possible in the presence of other people. VR users in the study were able to recall past events and particular points of interest. VR has been used as a tool to aid recovery from trauma by enabling viewers to confront and overcome bad memories. This research suggests that VR can also be used to do the opposite: to resurface and encourage the development of good memories. This provides an opportunity to use VR to temporally and geographically extend the experiencescape. By enabling viewers to recall moments of euphoria, it may build additional positive associations and stronger sentiment towards the event and its activities. This extended experiencescape can also be used to provide access to users who were not able to be physically present at the time of the activity.

\section{Conclusions and Recommendations}

The conventional view is that VR event experiences are currently not capable of substituting real event experiences, as the benefits do not exceed a real event (Adema \& Roehl, 2010). Others suggest that individuals will attend virtual events when the real event is unavailable (Getz, 2016). However, this research suggests that both perspectives are incomplete. Users may obtain sensory gratifications from virtual events even after they have seen the live act as a way of reliving the experience in private. In this way, positive experiences can be revisited and reinforced, strengthening sentiment or emotion towards a particular brand, sports team, or performer, increasing loyalty. Because events are episodic, this element may be of value to both researchers and practitioners. Future academic research can examine the role that VR can play in establishing and maintaining events brands. VR may play a role in developing a network identity that can relate potential customers to destinations (Williams \& Hristov, 2018).

Organizations may wish to determine the type and scale of VR that is appropriate for brand development. Further, content and design could provide users with opportunities to be active participants, not merely passive viewers. Since the equipment for $360^{\circ}$ capture is falling in price, there may be an opportunity for cocreation with attendees (Mathis, Kim, Uysal, Sirgy \& Prebensen, 2016.). Organizations may incentivize attendees to capture moments from a fan perspective that can be shared on social media. For organizations with a significant amount of resources, organizations can develop longer VR experiences with a guided narrative, which may increase the level of involvement and engagement.

An option could be presented to enable viewers to explore alternative, unscripted perspectives. A choice of viewpoint is one suggested method of introducing user interactions, which could strengthen feelings of presence (Carrillat, d'Astous, Bellavance, \& Eid, 2015; Stein, 2016). Comments 
or reactions from previous users could also be presented as a means of simulating a communal VR experience as distinct from an individual viewing of content.

Implementing a well-defined storyline could give users the context necessary to avoid confusion or disorientation. The VR event experience could also be designed to incorporate additional senses, which could result in a higher level of immersion and presence (Martins et al., 2017). During the stage of capturing VR content, camera equipment should be placed in natural consumer positions and avoid movement. Producers can also use "allin-one” filming equipment, such as the Jump 360 16-camera device (Anderson et al., 2016). These considerations could improve authenticity and increase a sense of presence. Additionally, creators should consider the intended sound design when capturing the live audio (Lelyveld, 2015). Highquality sound can be achieved through sophisticated audio recording equipment and high specification headphones, which could result in an increase in immersion. Future research can also examine the gratifications that are derived from sharing VR experiences. If VR can be used to encourage recall of specific memories, sharing of this content may provide a social memory gratification for both source and recipient.

\section{References}

Adema, K. L., \& Roehl, W. S. (2010). Environmental scanning the future of events. International Journal of Hospitality Management, 29(2), 199-207.

Anderson, R., Gallup, D., Barron, J. T., Kontkanen, J., Snavely, N., Hernández, C., ... \& Seitz, S. M. (2016). Jump: Virtual reality video. ACM Transactions on Graphics (TOG), 35(6), Article 198.

Arnould, E. J., \& Price. L. L. (1993). River magic: Extraordinary experience and the extended service encounter. Journal of Consumer Research, 20(1), 24-45.

Aylett, R., \& Louchart, S. (2003). Towards a narrative theory of virtual reality. Virtual Reality, 7, 2-9.

Ayob, N., Wahid, N., \& Omar, A. (2013b). Attributional model of visitors' event experience in festival and special events. International Journal of Event Management Research, 8(1), 21-32.

Barnes, S. J. (2017). Understanding virtual reality in marketing: Nature, implications and potential. Retrieved from https://papers.ssrn.com/sol3/Papers.cfm?abstract_id= 2909100
Bates-Brkljac, N. (2012). Virtual reality. New York, NY: Nova Science Publishers.

Benedek, J., \& Miner, T. (2002). Measuring desirability: New methods for evaluating desirability in a usability lab setting. Proceedings of Usability Professionals Association, 2003(8-12), 57.

Berridge, G. (2015). Event experiences: Design, management and impact (Ph.D. thesis). University of West London.

Bladen, C., Kennell, J., Abson, E., \& Wilde, N. (2012). Events management: An introduction. Abingdon, UK: Routledge.

Braun, B., \& Clarke, V. (2013). Successful qualitative research: A practical guide for beginners. London, UK: SAGE Publication Ltd.

Carlozzi, N. E., Gade, V., Rizzo, A., \& Tulsky, D. S. (2013). Using virtual reality driving simulators in persons with spinal cord injury: Three screen display versus head mounted display. Disability and Rehabilitation: Assistive Technology, 8(3), 176-180.

Carrillat, F., d’Astous, A., Bellavance, F., \& Eid, F. (2015). On 'being there': A comparison of the effectiveness of sporting event sponsorship among direct and indirect audiences. European Journal of Marketing, 49(3/4), 621-642.

Chen, P. J., Singh, D., Ozturk, A. B., \& Makki, A. (2014). Can fundraising be fun? An event management study of unique experiences, performance and quality. Tourism Review, 69(4), 310-328.

Chung, N., Han, H., \& Joun, Y. (2015). Tourists' intention to visit a destination: The role of augmented reality (AR) application for a heritage site. Computers in Human Behavior, 50, 588-599.

Dibbets, P., \& Schulte-Ostermann, M. A. (2015). Virtual reality, real emotions: A novel analogue for the assessment of risk factors of post-traumatic stress disorder. Frontiers in Psychology, 6(681), 1-8.

Disztinger, P., Schlögl S., \& Groth A. (2017). Technology acceptance of virtual reality for travel planning. In R. Schegg \& B. Stangl (Eds.), Information and communication technologies in tourism 2017. Cham, Switzerland: Springer.

Falconer, C. J., Slater, M., Rovira, A., King, J. A., Gilbert, P., Antley, A., \& Brewin, C. R. (2014). Embodying compassion: A virtual reality paradigm for overcoming excessive self-criticism. PLoS One, 9(11), e111933.

Ferdinand, N., \& Williams, N. L. (2018). The making of the London Notting Hill Carnival festivalscape: Politics and power and the Notting Hill Carnival. Tourism Management Perspectives, 27, 33-46.

Freeman, D., Antley, A., Ehlers, A., Dunn, G., Thompson, C., Vorontsova, N., ... \& Slater, M. (2014). The use of immersive virtual reality (VR) to predict the occurrence 6 months later of paranoid thinking and posttraumatic stress symptoms assessed by self-report and interviewer methods: A study of individuals who have been physically assaulted. Psychological Assessment, 26(3), 841-847. 
Frew, M. (2016). Future events and technology culture: Dreamscapes or dark fantasy? In D. Getz (Ed.), Event studies: Theory, research and policy for planned events (3rd ed.). London, UK: Routledge, Taylor \& Francis Group.

Getz, D. (2008). Event tourism: Definition, evolution, and research. Tourism Management, 29(3), 403-428.

Getz, D. (2012). Event studies: Discourses and future directions. Event Management, 16(2), 171-187.

Getz, D. (2016). Event studies: Theory, research and policy for planned events (3rd ed.). London, UK: Routledge, Taylor \& Francis Group.

Getz, D., \& Robinson, R. N. S. (2014). Foodies and food events. Scandinavian Journal of Hospitality and Tourism, 14(3), 315-330.

Geus, S. D., Richards, G., \& Toepoel, V. (2016). Conceptualisation and operationalisation of event and festival experiences: Creation of an event experience scale. Scandinavian Journal of Hospitality \& Tourism, 16(3), 274-296.

Gugenheimer, J., Wolf, D., Hass, G., Krebs, S., \& Rukzio, E. (2016). SwiVRChair: A motorized swivel chair to nudge users' orientation for 350 degree storytelling in virtual reality. In CHI '16 Proceedings of the 2017 CHI Conference on Human Factors in Computing Systems (pp. 1996-2000). New York, NY: Association for Computing Machinery.

Gutierrez, M., Vexo, F., \& Thalmann, D. (2008). Stepping into virtual reality. London, UK: Springer Science \& Business Media.

Han, H., Yoon, H., \& Woods, D. P. (2016). Role of environmental consciousness in golfers' decision formation in the traditional and screen-golf industries. Journal of Quality Assurance in Hospitality \& Tourism, 17(3), 290-310.

Haridakis, P. (2013). Uses and gratifications: A social and psychological perspective of media use and effects. In The international encyclopaedia of media studies. Oxford, UK: Wiley-Blackwell.

Howard, M. C. (2017). A meta-analysis and systematic literature review of virtual reality rehabilitation programs. Computers in Human Behavior, 70, 317-327.

Huang, Y. C., Backman, S. J., Backman, K. F., \& Moore, D. (2013). Exploring user acceptance of 3D virtual worlds in travel and tourism marketing. Tourism Management, 36, 490-501.

Kinateder, M., Ronchi, E., Nilsson, D., Kobes, M., Müller, M., Pauli, P., \& Mühlberger, A. (2014). Virtual reality for fire evacuation research. In Federated conference on computer science and information systems (FedCSIS) (Vol. 2, pp. 313-321). Retrieved from https://portal. research.lu.se/ws/files/3483362/4610536.pdf

Karapanos, E., Teixeira, P., \& Gouveia, R. (2016). Need fulfilment and experiences on social media: A case on Facebook and WhatsApp. Computers in Human Behaviour, 55, 888-897.

Kilteni, K., Grau-Sánchez, J., Veciana De Las Heras, M., Rodríguez-Fornells, A., \& Slater, M. (2016). Decreased corticospinal excitability after the illusion of missing part of the arm. Frontiers in Human Neuroscience, 10, 145.

Kuliga, S. F., Thrash, T., Dalton, R. C., \& Hölscher, C. (2015). Virtual reality as an empirical research toolExploring user experience in a real building and a corresponding virtual model. Computers, Environment and Urban Systems, 54, 363-375.

Kwon, J. H., Powell, J., \& Chalmers, A., (2013). How level of realism influences anxiety in virtual reality environments for a job interview. International Journal of Human-Computer Studies, 71(10), 978-987.

Lee, I., Brown, G., King, K., \& Shipway, R. (2016). Social identity in serious sport event space. Event Management, 20(4), 491-499.

Lelyveld, P. (2015). Virtual reality primer with an emphasis on camera-captured VR. SMPTE Motion Imaging Journal. Retrieved from http://www.etcenter.org/wp-content/ uploads/2015/07/ETC-VR-Primer-July-2015o.pdf

Lichtenstein, A., \& Rosenfeld, L. B. (1983). Uses and misuses of gratifications research: An explication of media functions. Communication Research, 10(1), 97-109.

Liu, W., Sparks, B., \& Coghlan, A. (2016). Measuring customer experience in situ: The link between appraisals, emotions and overall assessments. International Journal of Hospitality Management, 59, 42-49.

Maffei, L., Masullo, M., Pascale, A., Ruggiero, G., \& Romero, V. (2016). Immersive virtual reality in community planning: Acoustic and visual congruence of simulated vs real world. Sustainable Cities and Society, 27, 338-345.

Małgorzata, K. K. (2014). Consumers, play and communitas - An anthropological view on building consumer involvement on a mass scale. Polish Sociological Review, 3(187), 317-331.

Marchiori, E., Niforatos, E., \& Preto, L. (2017). Measuring the media effects of a tourism-related virtual reality experience using biophysical data. In R. Schegg \& B. Stangl (Eds.), Information and communication technologies in tourism 2017. Cham, Switzerland: Springer.

Martins, J., Gonçalves, R., Branco, F., Barbosa, L., \& Melo. M. (2017). A multisensory virtual experience model for thematic tourism: A Port wine tourism application proposal. Journal of Destination Marketing \& Management, 6(2), 103-109.

Mathis, E. F., Kim, H. L., Uysal, M., Sirgy, J. M., \& Prebensen, N. K. (2016). The effect of co-creation experience on outcome variable. Annals of Tourism Research, 57, 62-75.

Meinel, L., Heß, M., Findeisen, M., \& Hirtz, G. (2017). Effective display resolution of 360-degree video footage in virtual reality. In IEEE International Conference on Consumer Electronics (pp. 21-24). New York, NY: IEEE.

Mintel (2016a). Health and fitness clubs - UK - July 2016. London, UK: Author.

Mintel (2016b). Music concerts and festivals - UK-August 2016. London, UK: Author. 
Mintel (2016c). Digital trends - UK - October 2016. London, UK: Author.

Morgan, M. (2008). What makes a good festival? Understanding the event experience. Event Management, 12, 81-93.

Mueser, D., \& Vlachos, P. (2018). “Almost like being there?”: A conceptualisation of live-streaming theatre. International Journal of Event and Festival Management, 9(2), 183-203.

Murray, E., Neumann, D., Moffitt, R., \& Thomas, P. (2016). The effects of the presence of others during a rowing exercise in a virtual reality environment. Psychology of Sport \& Exercise, 22, 328-336.

Nasrabadi, A. T., Mahzari, A., Beshay, J., \& Prakash, R. (2017). Adaptive 360-degree video streaming using layered video coding. In 2017 IEEE virtual reality. New York, NY: IEEE.

Nordvall, A., Pettersson, R., Svensson, B. \& Brown, S. (2014). Designing events for social interactions. Event Management, 18(2), 127-140.

Palinkas, L. A., Hotwitz, S. M., Green, C. A., Wisdom, J. P., Duan, N., \& Hoagwood, K. (2015). Purposeful sampling for qualitative data collection and analysis in mixed method implementation research. Administration and Policy in Mental Health, 42(5), 533-544.

Papacharissi, Z. (2009). The citizen is the message: Alternative modes of civic engagement. In Journalism and citizenship: New agendas in communication (pp. 47-61). New York, NY: Routledge.

Peperkorn, H. M., Diemer, J., \& Mühlberger, A. (2015). Temporal dynamics in the relation between presence and fear in virtual reality. Computers in Human Behavior, 48, 542-547.

Pine, B., \& Gilmore, J. (1998). Welcome to the experience economy. Harvard Business Review, 76(4), 97-105.

Pope, V. C., Dawes, R., Scheweiger, F., \& Sheikh, A. (2017). The geometry of storytelling: Theatrical use of space for 360-degree videos in virtual reality. In CHI '17 Proceedings of the 2017 CHI conference on human factors in computing systems (pp. 4468-4478). New York, NY: Association for Computing Machinery.

Quan-Haase, A., \& Young, A.L. (2010). Uses and gratifications of social media: A comparison of Facebook and instant messaging. Bulletin of Science, Technology \& Society, 30(5), 350-361.

Rebelo, F., Noriega, P., Duarte, E., \& Soares, M. (2012). Using virtual reality to assess user experience. Human Factors, 54(6), 964-982.

Richards, G., Marques, L., \& Mein, K. (2014). Event design: Social perspectives and practices. London, UK: Routledge.

Richards, G., Marques, L., \& Mein, K. (2015). Event design: Social perspectives and practises. Abingdon, UK: Routledge.
Schmitt, B. (2003). Customer experience management: A revolutionary approach to connection with your customers. Hoboken, NJ: Wiley.

Serrano, B., Botella, C., Baños, R., \& Alcañiz, M. (2013). Using virtual reality and mood-induction procedures to test products with consumers of ceramic tiles. Computers in Human Behavior, 29(3), 648-653.

Sheldon, K. M., Elliot, A. J., Kim, Y., \& Kasser, T. (2001). What is satisfying about satisfying events? Testing 10 candidate psychological needs. Journal of Personality and Social Psychology, 80(2), 325.

Silver, C., \& Lewins, A., 2014. Using software in qualitative research. London, UK: SAGE Publications Ltd.

Slater, M., \& Sanchez-Vives, M. V. (2016). Enhancing our lives with immersive virtual reality. Frontiers in Robotics and AI, 3, Article 74.

Stein, C. (2016). Virtual reality design: How upcoming head-mounted displays change design paradigms of virtual reality worlds. Media Tropes, 6(1), 52-85.

Sundar, S. S. (2008). The MAIN model: A heuristic approach to understanding technology effects on credibility. In M. J. Metgzer \& A. J. Flanagin (Eds.), Digital media, youth, and credibility (pp. 73-100). Cambridge, MA: The MIT Press.

Sundar, S. S., \& Limperos, A. M. (2013). Uses and grats 2.0: New gratifications for new media. Journal of Broadcasting \& Electronic Media, 57(4), 504-525.

Swanson, D. L. (1992). Understanding audiences: Continuing contributions of gratifications research. Poetics, 21(4), 305-328.

tom Dieck, M. C., Jung, T. H., \& Rauschnabel, P.A. (2018). Determining visitor engagement through augmented reality at science festivals: An experience economy perspective. Computers in Human Behavior, 82, 44-53.

Treleaven, J., Battershill, J., Cole, D., Fadelli, C., Freestone, S., Lang, K., \& Sarig-Bahat, H. (2015). Simulator sickness incidence and susceptibility during neck motioncontrolled virtual reality tasks. Virtual Reality, 19(3/4), 267-275.

Tussyadiah, I. P., Wang, D., Jung, T. H., \& tom Dieck, M. C. (2018). Virtual reality, presence, and attitude change: Empirical evidence from tourism. Tourism Management, 66, 140-154.

Wan, C. S., Tsaur, S. H., Chiu, Y. L., \& Chiou, W. B. (2007). Is the advertising effect of virtual experience always better or contingent on different travel destinations? Information Technology \& Tourism, 9(1), 45-54.

Williams, N. L., \& Hristov, D. (2018). An examination of DMO network identity using Exponential Random Graph Models. Tourism Management, 68, 177-186.

Yung, R., \& Khoo-Lattimore, C. (2017). New realities: A systematic literature review on virtual reality and augmented reality in tourism research. Current Issues in Tourism. doi: 10.1080/13683500.2017.1417359. 